\title{
Lactobacillus Sepsis and Probiotic Therapy in Newborns: Two New Cases and Literature Review
}

\author{
Carlo Dani, MD ${ }^{1}$ Caterina Coviello C, MD ${ }^{2}$ luri Corsini I, MD ${ }^{2}$ Fabio Arena, MD \\ Alberto Antonelli, MD ${ }^{4}$ Gian Maria Rossolini, MD ${ }^{5}$
}

${ }^{1}$ Department of Neuroscience, Psychology, Drug Research and Child
Health, Careggi University Hospital of Florence, Florence, Italy
2 Division of Neonatology, Careggi University Hospital of Florence,
Florence, Italy
${ }^{3}$ Department of Medical Biotechnologies, University of Siena,
Siena, Italy
${ }^{4}$ Department of Experimental and Clinical Medicine, University of
Florence, Florence, Italy
${ }^{5}$ Clinical Microbiology and Virology Unit, Careggi University Hospital
of Florence, Florence, Italy

Address for correspondence Carlo Dani, MD, Division of Neonatology, Careggi University Hospital, Largo Brambilla, 3, 50141-Florence, Italy (e-mail: cdani@unifi.it).

Am J Perinatol Rep 2016;6:e25-e29.

\begin{abstract}
Keywords

- lactobacillus

- probiotics

- infection

- sepsis

- infant

Many term and preterm infants are commonly supplemented with probiotics to prevent adverse effects of antibiotic administration and necrotizing enterocolitis and they are believed to be safe. However, the supplementation with Lactobacillus rhamnosus GG has been associated with the development of sepsis with a cause-effect relationship in six newborns and children. In this study, we report two further cases and discuss the emerging issue of probiotic supplementation safety in neonates. We conclude that physicians must be aware that supplementation with $L$. rhamnosus GG can cause sepsis in high-risk patients on rare occasions.
\end{abstract}

It is well known that necrotizing enterocolitis $(\mathrm{NEC})^{1}$ and nosocomial infections ${ }^{2}$ increase morbidity and mortality in preterm infants and, therefore, their prevention is of crucial importance for improving outcome in these patients. Probiotic supplementation has been widely studied as one of the proposed interventions for the prophylaxis of NEC and nosocomial infections. Probiotics can enhance the enteric microbiota composition and counteract the loss of gut commensals such as Bifidobacterium and Lactobacillus species, as occurs in preterm infants undergoing prolonged antibiotic treatment, delayed enteral feeding, and lack of human milk, which can favor the proliferation of pathogenic microflora and abnormal gut colonization. ${ }^{3}$ Thus, probiotics may help to decrease translocations of pathogens from the gut and ultimately the development of NEC and nosocomial infections. ${ }^{3}$

A recent meta-analysis of 24 randomized controlled studies showed that probiotics are effective in significantly de- creasing NEC occurrence and mortality, but not nosocomial infections, and concludes that these findings support a change in the current practice and they should be widely used. ${ }^{4}$ It is noteworthy that this review specifies that none of the included studies report systemic infections due to administered probiotic organisms, thus supporting the safety of probiotic supplementations in preterm infants. ${ }^{4}$

On this basis, every day thousands of extremely and very preterm infants have been and are supplemented with probiotics. However, some cases of sepsis attributable to Lactobacillus species have been documented in patients supplemented with probiotics, such as two preterm infants with short-gut syndrome, ${ }^{5}$ one child with short-gut syndrome, ${ }^{5}$ one infant with congenital heart disease, ${ }^{6}$ one child with cerebral palsy, ${ }^{6}$ and one term infant with intrauterine growth restriction. ${ }^{7}$ Moreover, these reports confirm previous concerns regarding the risk of infections due to received

June 22, 2015

accepted after revision

August 20, 2015

published online

October 28, 2015
DOI http://dx.doi.org/

10.1055/s-0035-1566312. ISSN 2157-6998.
Copyright $\odot 2016$ by Thieme Medical Publishers, Inc., 333 Seventh Avenue, New York, NY 10001, USA. Tel: +1(212) 584-4662.
License terms

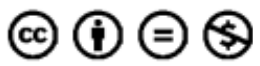


Lactobacillus species previously documented in adult human beings. $^{8,9}$

Thus, the purpose of this report is to document two further cases of sepsis caused by Lactobacillus rhamnosus that occurred in our neonatal intensive care in a term infant affected by multiple chromosomal disorders and in an extremely preterm infant, respectively, and to discuss the emerging issue of probiotic supplementation safety in neonates.

\section{Data Sources}

The National Library of Medicine (MEDLINE) database was searched from 1995 to 2014. Search criteria included the following MESH: (1) Lactobacillus or probiotic; (2) sepsis, bacteremia, or short-gut syndrome; and (3) infant, newborn, preterm, or premature.

\section{Case 1}

A Caucasian female was born at 39 weeks of gestation by vaginal delivery and was affected by trisomy 18 and triple-X syndromes. Her birthweight was 1,660 $\mathrm{g}$ and Apgar scores were 5 and 8 at 1 and 5 minutes, respectively. Heart ultrasound demonstrated atrial and ventricular septal defects, bicuspid aortic valve, and patent ductus arteriosus (PDA). Furthermore, her postnatal course was complicated by status epilepticus, relapsing systemic infections (sepsis caused by Staphylococcus aureus, pneumonia caused by Stenotrophomonas maltophilia and Staphylococcus aureus), respiratory failure requiring mechanical ventilation (MV), need of pulmonary arterial banding, and surgical closure of the PDA for hemodynamic worsening. On day 97 of life, during MV and with central venous catheter (CVC) in place, the patient had a temperature of $38.7^{\circ} \mathrm{C}$ and pulse of 120 beats/min, without other signs and symptoms. Blood sample for culture was drawn from CVC and a peripheral vein, bronchoalveolar lavage for culture was performed, and empiric antibiotic treatment with daptomycin ( $6 \mathrm{mg} / \mathrm{kg}$, dose every 24 hours) and ceftazidime (30 mg/kg, dose every 6 hours) was started. Laboratory analyses evidenced a white blood cell count (WBC) of 8,040 cells $/ \mathrm{mL}$, platelet count of 80,000 cells $/ \mathrm{mL}$, serum C-reactive protein (CRP) level of $135.3 \mathrm{mg} / \mathrm{L}$, and serum procalcitonin (PCT) level of $10.12 \mathrm{ng} / \mathrm{mL}$. Blood culture from the peripheral vein was positive for $L$. rhamnosus species. Since the 9th day of life our patient was given oral drop supplementation with $5 \times 10^{9}$ colony-forming unit (CFU) of L. rhamnosus GG (Dicoflor, Dicofarm, Rome, Italy) twice daily, through the orogastric tube, for the prevention antibioticassociated diarrhea. After the results of a positive blood culture the probiotic supplementation was discontinued. The isolate Lactobacillus isolate was susceptible to penicillin $\mathrm{G}$, erythromycin, ampicillin, gentamicin, clindamycin, linezolid, and was resistant to vancomycin. Therefore, we discontinued ceftazidime and started clindamycin $(5 \mathrm{mg} / \mathrm{kg}$, dose every 6 hours). Our patient's clinical conditions remained stable and after 10 days of therapy with clindamycin, her WBC, CRP, and PCT normalized and antibiotic therapy was discontinued. Ultimately, our patient was discharged at
300 days of life with gastrostomy for enteral nutrition and tracheotomy for respiratory support due to her syndromes.

\section{Case 2}

A white male, was born at 23 weeks of gestation by vaginal delivery. His birth weight was $660 \mathrm{~g}$ and Apgar scores were 4 and 7 at 1 and 5 minutes, respectively. The postnatal course was complicated by the development of respiratory distress syndrome, pharmacological closure of PDA, and sepsis caused by Staphylococcus haemolyticus. On the 18th day of life, during noninvasive respiratory support (nasal intermittent $\mathrm{MV}$ ) and with CVC in place, the patient developed episodes of mixed apnea associated with metabolic acidosis without other signs and symptoms of infection. Blood samples for culture were made from the CVC and a peripheral vein, and empiric antibiotic treatment with linezolid $(10 \mathrm{mg} / \mathrm{kg}$, dose every 8 hours) and gentamicin ( $4 \mathrm{mg} / \mathrm{kg}$, dose every 36 hours) was started. Laboratory analyses evidenced a WBC of 20,500 cells/mL, CRP of $25.7 \mathrm{mg} / \mathrm{L}$, and PCT of $2.90 \mathrm{ng} / \mathrm{mL}$. The blood culture from the peripheral vein was positive for L. rhamnosus. Since the 2 nd day of life our patient was given daily oral drop supplementation with $5 \times 10^{9} \mathrm{CFU}$ of $L$. rhamnosus GG (Dicoflor), through the orogastric tube, to prevent NEC. After positive blood culture appeared, probiotic supplementation was discontinued. The isolate had the same antibiotic susceptibility and resistance of the previous case. Therefore, we discontinued gentamicin and started clindamycin $(5 \mathrm{mg} / \mathrm{kg}$, dose every 6 hours) that was given for 10 days.

On the 26th day of life, the infant developed severe respiratory failure requiring MV and $100 \%$ oxygen, caused by a chest Xray confirmed pneumonia. Laboratory analyses evidenced a WBC of 6,950 cells/mL, platelet count of 119,000 cells/mL, CRP of $84.7 \mathrm{mg} / \mathrm{L}$, and PCT of $3.80 \mathrm{ng} / \mathrm{mL}$. Blood samples for culture were made from the CVC and a peripheral vein, and daptomycin ( $6 \mathrm{mg} / \mathrm{kg}$, dose every 24 hours) and meropenem ( $20 \mathrm{mg} / \mathrm{kg}$, dose every 8 hours) were administered empirically. Also, the second peripheral blood culture was positive for L. rhamnosus with the same antimicrobial susceptibility profile as the first positive blood culture and therefore meropenem was stopped and gentamicin ( $4 \mathrm{mg} / \mathrm{kg}$, dose every 24 hours) was given. Persistence of $L$. rhamnosus bacteremia was documented in a third blood sample, obtained from a peripheral vein after 6 days (34th day of life). However, after 10 days of therapy with gentamicin, WBC, CRP, PCT, and chest X-ray normalized, and his clinical condition progressively improved. Ultimately, our patient was discharged at 117 days of life in good health.

\section{Characterization of the Lactobacillus Isolates}

The Lactobacillus isolates from the two blood cultures were identified by MALDI-TOF mass spectrometry (VITEKMS, bioMérieux, Marcy L'Etoil, France) as L. rhamnosus, suggesting a correlation with the probiotic preparation given to the infants. To compare the two cultures with the probiotic strain, genotyping by pulsed field gel electrophoresis (PFGE) profiling of the genomic DNA digested with the NotI and SfiI restriction endonucleases was performed. 
Table 1 Comparison of MIC values (determined by broth microdilution method) of penicillin, erythromycin, ampicillin, gentamycin, clindamycin, linezolid, and vancomycin of the two Lactobacillus rhamnosus clinical isolates and L. rhamnosus GG. Results were interpreted according to CLSI M45-A2 document

\begin{tabular}{|l|l|l|l|l|l|l|}
\hline & Isolate case 1 & Interpretation & Isolate case 2 & Interpretation & $\begin{array}{l}\text { Lactobacillus } \\
\text { rhamnosus GG }\end{array}$ & Interpretation \\
\hline Penicillin & 0.5 & $\mathrm{~S}$ & 1 & $\mathrm{~S}$ & 0.5 & $\mathrm{~S}$ \\
\hline Erythromycin & $\leq 0.12$ & $\mathrm{~S}$ & $\leq 0.12$ & $\mathrm{~S}$ & $\leq 0.12$ & $\mathrm{~S}$ \\
\hline Ampicillin & 2 & $\mathrm{~S}$ & 1 & $\mathrm{~S}$ & 1 & $\mathrm{~S}$ \\
\hline Gentamicin & 4 & $\mathrm{~S}$ & 2 & $\mathrm{~S}$ & 4 & $\mathrm{~S}$ \\
\hline Clindamycin & $\leq 0.12$ & $\mathrm{~S}$ & $\leq 0.12$ & $\mathrm{~S}$ & $\leq 0.12$ & $\mathrm{~S}$ \\
\hline Linezolid & 2 & $\mathrm{~S}$ & 2 & $\mathrm{~S}$ & 1 & $\mathrm{~S}$ \\
\hline Vancomycin & $>256$ & $\mathrm{R}$ & $>256$ & $\mathrm{R}$ & $>256$ & $\mathrm{R}$ \\
\hline
\end{tabular}

Abbreviations: MIC, minimum inhibitory concentration, expressed in $\mu \mathrm{g} / \mathrm{mL}$; R, resistant; $\mathrm{S}$, susceptible.

The genomic DNA in agarose blocks was prepared by the method of Tynkkynen et al. ${ }^{10}$ Restriction enzyme digestion was performed overnight at $37^{\circ} \mathrm{C}$. Electrophoresis was performed with a CHEF-DRIII apparatus (Bio-Rad, Hemel Hempstead, United Kingdom) in 1\% PFGE certified agarose (BioRad) with $0.5 \times$ TBE buffer. The pulse time was 1 to 15 seconds the current was $5 \mathrm{~V} / \mathrm{cm}$, the temperature was $14^{\circ} \mathrm{C}$, and running time was 22 hours. After electrophoresis, the agarose gel was stained with ethidium bromide $(0.5 \mu \mathrm{g} / \mathrm{mL})$, visualized under ultraviolet light, and the PFGE profiles were compared.

The isolates from both the patients exhibited an identical PFGE profile to that of the probiotic strain L. rhamnosus GG (ATCC 53103) (data not shown). Antimicrobial susceptibility testing of the $L$. rhamnosus GG strain from the probiotic formulation revealed a profile identical to that of the two clinical isolates, with minimal inhibitory concentrations of penicillin $\mathrm{G}$, erythromycin, ampicillin, gentamicin, clindamycin, linezolid, daptomycin, and vancomycin of $0.5, \leq 0.12,1$, 4 , $\leq 0.12,1,1$, and $>256 \mathrm{mg} / \mathrm{L}$, respectively (-Table 1).

\section{Discussion}

In this study, we report two cases of sepsis caused by L. rhamnosus GG that developed during the patients' probiotic supplementation with the same strain, thus supporting a cause-effect relationship between supplementation and the development of sepsis. By reviewing the international literature we identified six other cases of sepsis due to L. rhamnosus GG occurring during probiotic supplementation with the same strain in infants ${ }^{5,7,8}$ and children ${ }^{6,7}$ (-Table 2). All these patients were supplemented with L. rhamnosus GG with the purpose of preventing or treating gastrointestinal complications, such as antibiotic-associated diarrhea or NEC.

These cases are in agreement with previous studies reporting the development of systemic infections caused by Lactobacillus species in infants and children who were not supplemented with probiotics. ${ }^{11-17}$ Both supplemented and nonsupplemented patients had similar risk factors, such as immune-deficiency (including that associated with prematu- rity $^{18}$ ), previous gastrointestinal or cardiac surgery, previous antibiotic therapy, particularly with vancomycin, NEC, ileostomy, malabsorption, and placement of CVC, but it is probable that supplementation may further enhance the risk of developing $L$. rhamnosus GG sepsis through the daily prolonged overload of microorganisms.

Thus, L. rhamnosus GG is considered a commensal microbe in human beings and part of the normal gut microbial flora, ${ }^{6}$ is safe and nonpathogenic in most patients, ${ }^{19}$ but can induce serious infections, including sepsis, ${ }^{5-8}$ pneumonia, and meningitis $^{14,15}$ in compromised newborns and children. It is likely that similar considerations may be extended to other probiotics commonly given to preterm infants, such as Bifidobacterium species, since five cases of bacteremia/sepsis have already been documented in newborns. ${ }^{20-22}$ However, it must be underlined that only a few cases of severe infection by probiotics have been reported in comparison to the thousands of preterm infants who have been or are supplemented for preventing NEC.

The pathogenesis of Lactobacillus infection is not well known, but its adhesion to the intestinal mucosa and subsequent colonization are considered important steps because they can prolong persistence in the intestine. ${ }^{23}$ This consideration seems to support our speculation that prolonged daily probiotic supplementation, as occurred in our and previous patients, ${ }^{5-8}$ may represent a relevant risk factor for the development of related infections. When supplemented patients develop L. rhamnosus GG sepsis its only plausible portal of entry is through enteral administration that is probably followed by Lactobacillus access to the bloodstream through translocation across the epithelium. This event might be favored by local gut injuries, such as those potentially caused by decreased blood perfusion able to injure the gastrointestinal mucosa (i.e., systemic hypotension, gastrointestinal surgery, congenital heart disease, intrauterine growth restriction, treatment with nonsteroidal anti-inflammatory drugs for PDA closure, treatment with corticosteroids, etc.). Another uncommon possibility might be CVC contamination, either during the opening of the probiotic bottle or through hand-related transmission. 6 


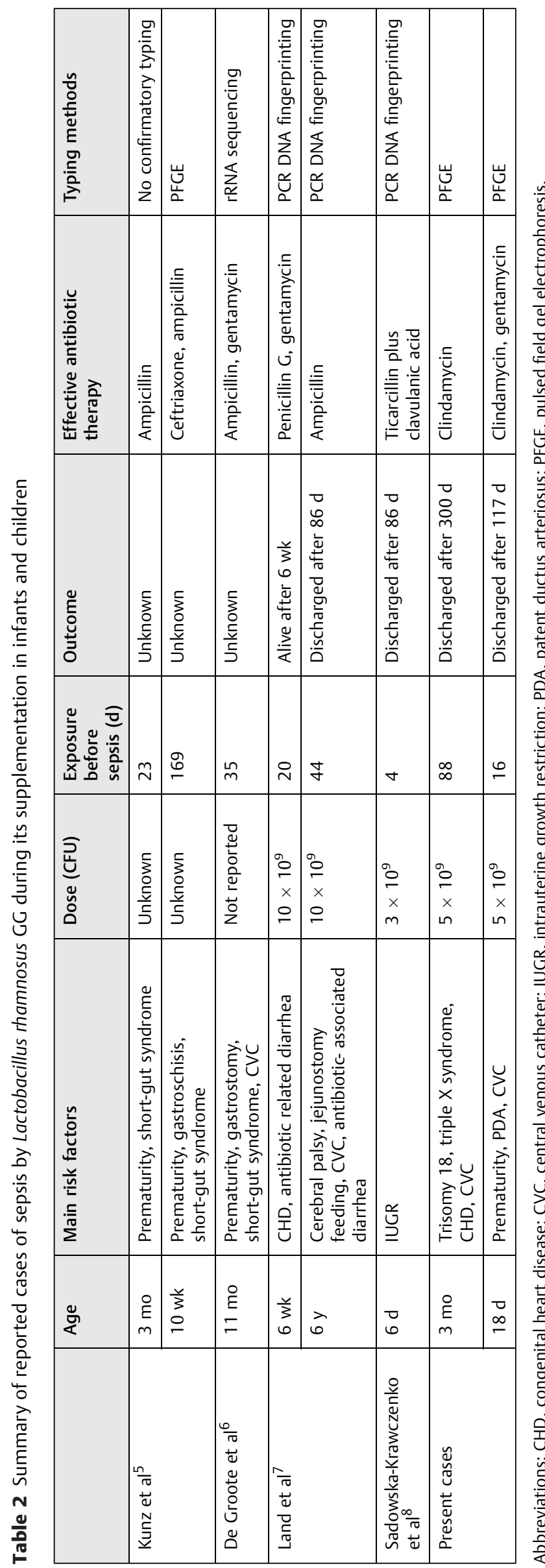

We evaluated the possible role of dose and duration of exposure to L. rhamnosus GG in our cases in comparison with previous reports, ${ }^{4-7}$ and we observed a great heterogeneity. In fact, while some reports did not detail the supplementation dose, ${ }^{4,5}$ we administered $10 \times 10^{9} \mathrm{CFU}$ in the first case and $5 \times 10^{9} \mathrm{CFU}$ in the second, Land et $\mathrm{al}^{7}$ gave $10 \times 10^{9} \mathrm{CFU}$, and Sadowska-Krawczenko et $\mathrm{al}^{7}$ gave $3 \times 10^{9} \mathrm{CFU}$. Moreover, the duration of supplementation with $L$. rhamnosus GG ranged from 4 to 95 days. ${ }^{5,8}$ Such heterogeneity precludes the possibility of drawing conclusions regarding the possible effect of probiotic dose and exposure duration on the risk of developing related sepsis. However, after these two cases and due to the lack of evidence-based recommendations on these points, ${ }^{24}$ we have decided to decrease the daily dose of L. rhamnosus GG in our patients to $3 \times 10^{9} \mathrm{CFU}$.

Antimicrobial susceptibility of the infecting $L$. rhamnosus GG strains has been reported in only some of the L. rhamnosus case reports reviewed in this article. ${ }^{5,7,8}$ These data showed some variability, but these discrepancies could also be attributed to differences among susceptibility testing techniques and interpretative criteria adopted by different laboratories. However, a consistent finding among all the reports was the resistance of $L$. rhamnosus strains to vancomycin and their susceptibility to ampicillin. ${ }^{5,7,8}$

In summary, we report two cases of sepsis in neonates caused by $L$. rhamnosus GG during enteral supplementation in addition to the six cases previously reported. ${ }^{5-8}$ Probiotic supplementation most likely caused the sepsis in these patients, although all of them had further documented risk factors for sepsis. In these few cases, the dose and duration of probiotic supplementation do not seem to be positively related to the risk of developing sepsis and the antibiotic susceptibility of isolated strains varied between patients. We conclude that, although none of the thousands of patients enrolled in previous studies ${ }^{4}$ developed systemic infections due to administered probiotics, neonatologists must be aware that supplementation with $L$. rhamnosus GG can cause sepsis in high-risk patients on rare occasions. Further studies evaluating the most effective and safe dose and duration of each probiotic supplementation should be performed.

\section{References}

1 Henry MC, Moss RL. Necrotizing enterocolitis. Annu Rev Med 2009;60:111-124

2 Didier C, Streicher MP, Chognot D, et al. Late-onset neonatal infections: incidences and pathogens in the era of antenatal antibiotics. Eur J Pediatr 2012;171(4):681-687

3 Manzoni P, De Luca D, Stronati M, et al. Prevention of nosocomial infections in neonatal intensive care units. Am J Perinatol 2013; 30(2):81-88

4 AlFaleh K, Anabrees J. Probiotics for prevention of necrotizing enterocolitis in preterm infants. Evid Based Child Health 2014;9(3):584-671

5 Kunz AN, Noel JM, Fairchok MP. Two cases of Lactobacillus bacteremia during probiotic treatment of short gut syndrome. J Pediatr Gastroenterol Nutr 2004;38(4):457-458

6 De Groote MA, Frank DN, Dowell E, Glode MP, Pace NR. Lactobacillus rhamnosus GG bacteremia associated with probiotic use in a child with short gut syndrome. Pediatr Infect Dis J 2005;24(3): 278-280 
7 Land MH, Rouster-Stevens K, Woods CR, Cannon ML, Cnota J, Shetty AK. Lactobacillus sepsis associated with probiotic therapy. Pediatrics 2005;115(1):178-181

8 Sadowska-Krawczenko I, Paprzycka M, Korbal P, et al. Lactobacillus rhamnosus GG suspected infection in a newborn with intrauterine growth restriction. Benef Microbes 2014;5(4):397-402

9 Cannon JP, Lee TA, Bolanos JT, Danziger LH. Pathogenic relevance of Lactobacillus: a retrospective review of over 200 cases. Eur J Clin Microbiol Infect Dis 2005;24(1):31-40

10 Tynkkynen S, Satokari R, Saarela M, Mattila-Sandholm T, Saxelin M. Comparison of ribotyping, randomly amplified polymorphic DNA analysis, and pulsed-field gel electrophoresis in typing of Lactobacillus rhamnosus and L. casei strains. Appl Environ Microbiol 1999;65(9):3908-3914

11 Bayer AS, Chow AW, Betts D, Guze LB. Lactobacillemia-report of nine cases. Important clinical and therapeutic considerations. Am J Med 1978;64(5):808-813

12 Schlegel L, Lemerle S, Geslin P. Lactobacillus species as opportunistic pathogens in immunocompromised patients. Eur J Clin Microbiol Infect Dis 1998;17(12):887-888

13 Kalima P, Masterton RG, Roddie PH, Thomas AE. Lactobacillus rhamnosus infection in a child following bone marrow transplant. J Infect 1996;32(2):165-167

14 Thompson C, McCarter YS, Krause PJ, Herson VC. Lactobacillus acidophilus sepsis in a neonate. J Perinatol 2001;21(4):258-260

15 Broughton RA, Gruber WC, Haffar AA, Baker CJ. Neonatal meningitis due to Lactobacillus. Pediatr Infect Dis 1983;2(5):382-384
16 Sriskandan S, Lacey S, Fischer L. Isolation of vancomycin-resistant lactobacilli from three neutropenic patients with pneumonia. Eur J Clin Microbiol Infect Dis 1993;12(8):649-650

17 Brook I. Isolation of non-sporing anaerobic rods from infections in children. J Med Microbiol 1996;45(1):21-26

18 Schelonka RL, Infante AJ. Neonatal immunology. Semin Perinatol 1998;22(1):2-14

19 Salminen MK, Tynkkynen S, Rautelin H, et al. Lactobacillus bacteremia during a rapid increase in probiotic use of Lactobacillus rhamnosus GG in Finland. Clin Infect Dis 2002;35(10):1155-1160

20 Ohishi A, Takahashi S, Ito Y, et al. Bifidobacterium septicemia associated with postoperative probiotic therapy in a neonate with omphalocele. J Pediatr 2010;156(4):679-681

21 Jenke A, Ruf EM, Hoppe T, Heldmann M, Wirth S. Bifidobacterium septicaemia in an extremely low-birthweight infant under probiotic therapy. Arch Dis Child Fetal Neonatal Ed 2012;97(3):F217-F218

22 Zbinden A, Zbinden R, Berger C, Arlettaz R. Case series of Bifidobacterium longum bacteremia in three preterm infants on probiotic therapy. Neonatology 2015;107(1):56-59

23 Saarela M, Mogensen G, Fondén R, Mättö J, Mattila-Sandholm T. Probiotic bacteria: safety, functional and technological properties. J Biotechnol 2000;84(3):197-215

24 Agostoni C, Buonocore G, Carnielli VP, et al; ESPGHAN Committee on Nutrition. Enteral nutrient supply for preterm infants: commentary from the European Society of Paediatric Gastroenterology, Hepatology and Nutrition Committee on Nutrition. J Pediatr Gastroenterol Nutr 2010;50(1):85-91 